\title{
The effect of dose on resistance exercise therapies for tendinopathy: A systematic review and meta-analysis protocol.
}

Swinton, P.A. ${ }^{*}$ Shim, J. ${ }^{1}$ Pavlova, A.V. ${ }^{1}$ Moss, R.A. ${ }^{1}$ Maclean, C. ${ }^{2}$ Brandie, D. ${ }^{3}$ Mitchell, L. ${ }^{4}$ Greig, L. ${ }^{1}$ Parkinson, E. ${ }^{1}$ Morrissey, D. ${ }^{5}$ Alexander, L. ${ }^{1}$ Cooper, K. ${ }^{1}$

\begin{tabular}{|c|}
\hline DOI: $10.31236 /$ osf.io/an3j6 \\
SportR $\chi$ iv hosted preprint version 1 \\
Last updated \\
PREPRINT - NOT PEER REVIEWED
\end{tabular}

Institutions

${ }^{1}$ School of Health Sciences, Robert Gordon University, Aberdeen, UK

2 Library Services, Robert Gordon University, Aberdeen, UK

${ }^{3}$ Sport Scotland, Inverness, UK

${ }^{4}$ NHS Grampian, Aberdeen, UK

${ }^{5}$ Centre for Sports \& Exercise Medicine, Barts \& the London School of Medicine \& Dentistry, London, UK

* Corresponding Author

Dr. Paul Swinton

School of Health Sciences, Robert Gordon University

Garthdee Road

Aberdeen, UK,

AB10 7QG

p.swinton@,rgu.ac.uk, +44 (0) 12242623361

\section{Funding}

This project is funded by the National Institute for Health Research (NIHR) [Health Technology Assessment (HTA) 129388 Exercise therapy for the treatment of tendinopathies]. The views expressed are those of the authors and not necessarily those of the NIHR or the Department of Health and Social Care.

\section{Conflicts of interest}

The authors declare no conflict of interest. 


\subsection{Introduction}

Tendinopathy is a common musculoskeletal condition associated with degenerative changes within a tendon affecting both athletic and non-athletic populations. ${ }^{1}$ The condition is characterized by a combination of pain, ${ }^{1}$ and impaired movement ${ }^{2}$ and function ${ }^{3}$, requiring extended periods for recovery. ${ }^{2,4-5}$ Tendinopathy can affect any muscle-tendon unit in the body, ${ }^{6}$ however, it is most frequently reported in the Achilles, patellar, lateral elbow, rotator cuff, and hip tendons. ${ }^{6}$ Surveys of prevalence of lower extremity tendinopathy in the general population have reported rates of 11.8 and 10.5 per 1000 person-years, ${ }^{7}$ whilst prevalence for upper limb tendinopathies have been estimated between 1.3\% to 21.0\%. ${ }^{8-10}$ Tendinopathies can affect children, adolescents, and adults of all ages, and many tendinopathies have a chronic or recurrent course. ${ }^{6}$ Costs to the individual, the health service and economy (due to absenteeism and loss of productivity) are substantial such that identifying effective interventions is a priority. Musculoskeletal conditions including tendinopathies also have a substantive influence on primary and secondary healthcare use. ${ }^{11}$ By identifying effective interventions across a range of tendinopathies, General Practitioners and other first-contact practitioners (e.g. physiotherapists) can be confident in delivering effective evidence-based practice. With an ageing population, and increasing pressure and demands on healthcare services, the need for clear guidance for evidence-based practice has never been more important.

Exercise therapy is the mainstay of conservative management of tendinopathy and has focused largely on resistance training, and in many instances eccentric strengthening techniques, to date. ${ }^{12}$ The objective with exercise therapy is to encourage load tolerance that leads to structural adaptation at the musculotendinous unit and restores function. ${ }^{13-14}$ Isometric, isotonic, and heavy slow resistance training have also been recommended for some tendinopathies (e.g. patellar) with suggested efficacy. ${ }^{15}$ The effectiveness of exercise therapy is likely to be influenced not only by the specific exercises performed but also the magnitude of the stimulus described by the concept of exercise dose. ${ }^{16}$ At the most basic level in clinical settings, exercise dose comprises three variables including frequency, duration, and intensity, with overall exercise dose quantified as the product of all three variables. ${ }^{17}$ With an increasing evidence base of effectiveness across a range of populations and tendinopathies, it has been recommended that both primary research and evidence synthesis studies attempt to identify dose-response relationships and ultimately seek to determine optimum exercise dosages. ${ }^{16,18,19}$ The potential to develop dose-response relationships may be most likely for resistance exercise due to the amount of data available from primary studies and the ability to accurately quantify dose variables including intensity. Initial attempts to synthesise evidence and identify dose-response relationships were limited by setting restrictive inclusion criteria substantially reducing the amount of data available. Meyer et al. ${ }^{19}$ only included three studies when investigating the effect of eccentric exercise protocols for Achilles tendinopathy. In a similar proceeding systematic review of eccentric exercise and Achilles tendinopathy, the number of included studies was increased to eight, however, the authors still concluded that heterogenous outcomes and methodological limitations meant that data could not be pooled, nor recommendations made regarding dose-response. ${ }^{20}$

Two recent approaches have been adopted in evidence synthesis research to better determine dose-response Swinton, Shim, Pavlova, Moss, Maclean, Greig, Parkinson, Morrissey, Alexander, Cooper (2021) 
DOI | SportsRxiv Preprint version 1

relationships in exercise therapy for tendinopathy. In a systematic review conducted by Naunton et al. ${ }^{21}$, pairwise effect sizes were calculated for studies that compared exercise therapies with placebo or no treatment arms in rotator cuff related shoulder pain. The approach enabled calculation of a relative effect size metric that could be pooled for levels of an exercise dose independently (e.g. low intensity vs high intensity), and then compared with each other. Naunton et al. ${ }^{21}$ used this approach to compare progressive resistance exercise with non-progressive resistance exercise. The systematic review identified four studies including progressive stimuli, and four studies that maintained a constant resistance exercise stimuli. Using measures of pain as a meta-analysis outcome, clear statistical evidence was obtained for a positive effect of progressive resistance exercise with best estimates identifying decreases in pain of between 10 and 15\%. In contrast, best estimates for decreases in pain were only between 1 and 3\% for non-progressive resistance exercise and all confidence intervals overstretched the regions of no effect and an effect favouring control. A second approach to investigate the effects of exercise dose was adopted by Young et al. ${ }^{16}$ who attempted to increase the amount of data by including research studying multiple common disorders. The systematic review and meta-analysis included eighteen studies across interventions investigating Achilles tendinopathy, ankle sprains and planar heel pain. Several trends were identified including greater effects with increased frequency and progressive exercise as tolerated compared with pre-prescribed sets and repetitions. However, no formal statistical comparisons of exercise dose were made by Young et al. ${ }^{16}$ limiting the conclusions that can be drawn.

The purpose of the present systematic review and meta-analysis is to investigate the effect of resistance exercise dose across multiple common tendinopathies (rotator cuff, lateral elbow, patellar or Achilles) where the frequency, volume and intensity can be accurately quantified. By combining a large data set with contemporary meta-analysis and meta-regression approaches (including relevant covariates within models), the present systematic review will attempt to explore statistical heterogeneity and better assess potential dose-response relationships that may exist. Where placebo and no-treatment arms are included, these studies will be used to reduce heterogeneity and provide sensitivity analyses to support or refute analyses with larger, but more complex data.

\subsection{Inclusion criteria}

This review is part of a project funded by the National Institute for Health Research (NIHR); Health Technology Assessment (HTA) 129388 Exercise therapy for the treatment of tendinopathies. The inclusion criteria are influenced by the project aims, the results of our initial scoping review mapping the exercise and tendinopathy literature 22 as well as stakeholder workshops.

\section{Participants}

This review will include people of any age or gender with a diagnosis of rotator cuff, lateral elbow, patellar or Achilles tendinopathy of any severity or duration. Studies that include participants with tendinopathy in the absence of full thickness or large tears, will be included. Groups where the tear size cannot be determined will also be excluded as these require different management approaches. We will accept trial authors' diagnoses where 
a clearly verifiable group of clinical features is reported including: pathognomonic location of pain; a symptom altering response to applied load and/or stretch, with there being a specific test for most tendinopathies; strategies to rule out differential diagnoses; ultrasound or magnetic resonance imaging confirmation of structural change. Studies with mixed groups will have data included where there is clear reporting of the tendinopathic group, or they make up $>90 \%$ of the investigated cohort.

\section{Intervention}

The health technology being assessed is resistance exercise which can be subcategorised based on the predominant contraction mode (see appendix I for definitions). Interventions combining resistance exercise with other active therapies (e.g. laser, shockwave, manual therapy or injection) will not be included. Resistance exercise may be delivered in a range of settings (e.g. primary care, secondary care, community, people's homes) by a range of health or exercise professionals (e.g. physiotherapists, strength \& conditioning coaches, personal trainers) or support workers, and may be supervised or unsupervised (i.e. self-management). No restrictions will be placed on these factors for inclusion. To be included in the review, studies are required to report sufficient information regarding the resistance exercise dose, including frequency (defined as the number of training sessions performed per week), volume (defined as the number of repetitions, sets, or repetitions multiplied by sets) and intensity (defined in absolute terms as the magnitude of the resistance used, or in relative terms either as a percentage of the maximum resistance that can be overcome for a single repetition or scaled to the maximum number of repetitions that can completed at a given absolute load). Where sufficient information is not presented in the main text of a study to quantify all three dose variables, a search will be made of the publishers' website to check for supplementary files that may include relevant information.

\section{Comparator}

Both non-controlled (resistance exercise only) and controlled (comparator adjusted) effects will be calculated. The comparator used for controlled effects will include placebo interventions and no treatment.

Outcomes

Based on the results of our initial scoping review and subsequent stake holder workshops we will include outcomes that assess five domains: 1) Disability; 2) Physical function capacity; 3) Pain on loading/activity; 4) Pain over a specified time; and 5) Pain without further specification. Definitions for each domain and example tools are presented in appendix II.

\section{Types of studies}

We will include randomized controlled trials and non-randomized controlled trials.

\section{Context}

The context will include primary care, secondary care or community locations in any developed nation (defined as the top 62 countries in the Human Development Index at the time of protocol development ${ }^{23}$ for the findings to be relevant to the UK context. 


\subsection{Exclusion criteria}

We will exclude self-described pilot studies and non-intervention studies where the purpose of the research is to investigate the acute effects of exercise on physiological or biomechanical variables such as pain, collagen turnover or mechanical properties of tendons.

\subsection{Methods}

Search strategy

The search strategy used for this study was part of a larger search conducted to scope the entire tendinopathy and exercise therapy research base. The search comprised three steps; Firstly, a limited search of MEDLINE and CINAHL using initial keywords (MH tendinopathy OR TX tendin* OR TX tendon*) AND (MH exercise OR TX exercis*) was conducted with analysis of the text words in the titles/abstracts and those used to describe articles to develop a full search strategy. Secondly, the full search strategy was adapted to each database and applied systematically to: MEDLINE, CINAHL, AMED, EMBase, SPORTDiscus, Cochrane library (Controlled trials, Systematic reviews), JBI Evidence Synthesis, PEDRo, and Epistemonikos (a full search strategy for MEDLINE is presented in appendix III). The following trial registries were also searched: ClinicalTrials.gov, ISRCTN Registry, The Research Registry, EU-CTR (European Union Clinical trials Registry), ANZCTR (Australia and New Zealand Clinical trials Registry). Finally, the third step involved conducting a search of cited and citing articles using Scopus and hand-searching a total of 130 systematic reviews that were identified to include information relevant to exercise therapy and tendinopathy. No limit was placed on language, with research studies published in languages other than English translated via Google Translate or via international collaborations of the review team members. Searches were initiated from 1998 as (i) the heavy load eccentric calf-training protocol for Achilles tendinosis by Alfredsson et al ${ }^{24}$ was published in 1998 and may be considered seminal work in the field of tendinopathy, and (ii) there has been a proliferation of research on exercise interventions for tendinopathies post 1998.

Study selection

Proquest ${ }^{\circledR}$ Refworks will be used to manage references and remove duplicates, before importing to Covidence (Melbourne, Australia) to facilitate screening. Two levels of screening will be conducted. First all titles/abstracts will be reviewed, independently, by two members of the research team. Conflicts will be resolved by discussion or by input from a third reviewer. Full-text copies of all studies included at title/abstract screening stage will be retrieved and these will also be screened independently by two members of the research team with conflicts resolved in the same way.

\section{Data extraction}

Data will be extracted independently by 8 members of the review team (PS/KC/LA/RM/LG/EP/JS/AP) into pre-piloted excel sheets. Data will be independently coded as described in the accompanying codebook Swinton, Shim, Pavlova, Moss, Maclean, Greig, Parkinson, Morrissey, Alexander, Cooper (2021) 
(appendix IV). To quantify reliability, $10 \%$ of studies will be selected at random and extraction completed in duplicate. Reliability will be quantified using Cohens $\mathrm{K}$ statistic ${ }^{25}$ for categorical variables and percentage agreement for continuous variables.

Risk of bias assessment

We will use the Cochrane Collaboration's Risk of Bias tool 26 and all five domains: 1) selection bias; 2) performance bias; 3) detection bias; 4) attrition bias; and 5) reporting bias, to assess risk of bias for all included RCTs. For non-random designs, we will use the ROBINS-I tool ${ }^{27}$ and all seven domains: 1) bias due to confounding; 2) bias in selection of participants into the study; 3) bias in classification of interventions; 4) bias due to deviations form intended interventions; and 5) bias due to missing data; 6) bias in measurement of outcomes; and 7) bias in selection of the reported. An overall risk of bias judgement will be made for each outcome and time point as either 'low risk', 'some concerns' or 'high risk' of bias. A single assessment will be made by a reviewer from the team with comments saved to justify selection for each signalling question. To quantify reliability, $10 \%$ of studies will be selected at random and extraction completed in duplicate.

\section{Statistical analysis}

We will fit treatment-level Bayesian models with standardised mean difference ( $\left.\mathrm{SMD}_{\mathrm{pre}}\right)$ effect sizes calculated by dividing the relevant mean difference by the pre-intervention standard deviation. As standard, non-controlled effect sizes will be calculated by subtracting baseline values from measurements made at subsequent time-points. Where placebo interventions or no treatment arms are included, the mean difference in the comparator will be subtracted from the mean difference in the resistance exercise intervention. Values will then be standardized by dividing by the pooled baseline standard deviation. Where sufficient data is presented for a single measurement tool, non-standardized effect sizes will also be included to facilitate clinical interpretations. To assess the effects of dose variables, meta-regressions will be performed with continuous covariates where appropriate spread of values are obtained, or where values are clustered binary or trinary categorisations will be made. Meta-regressions will only be performed where data from 10 or more trials are available for covariates or 4 or more trials per level for categorical variables.

Where outcomes are assessed at multiple time-points following baseline measurement, all possible $\mathrm{SMD}_{\text {pre values }}$

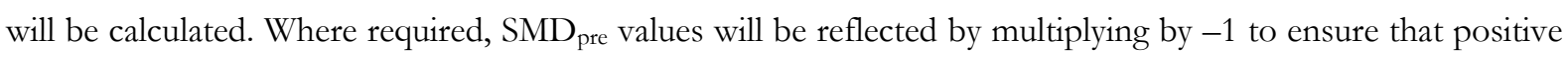
values represent a positive clinical effect and one that favours resistance exercise. Where baseline standard deviation values are not presented these will be estimated using statistical information presented ${ }^{28}$ (e.g. confidence intervals, standard errors, t values, P values, F values) or will be imputed through simple linear regression of the log-transformed standard deviations and means from studies included in the same analysis. ${ }^{29}$ Three-level Bayesian hierarchical models will be conducted to account for covariances in reporting of multiple

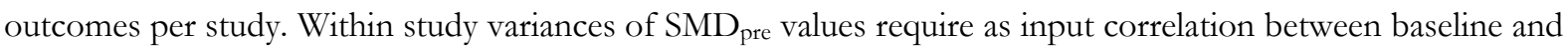
follow-up measurements. As this value is generally not presented in studies, informative priors centred on a correlation value of 0.5 will be included. Weakly informative Student-t prior and half-t priors with 3 degrees of freedom and scale parameter equal to 2.5 will be used for intercept and variance parameters (25). Inferences from all analyses will be performed on posterior samples generated by Markov Chain Monte Carlo simulations 
DOI | SportsR $x$ iv Preprint version 1

and through use of credible intervals and calculated probabilities. Analyses will be performed using the $\mathrm{R}$ wrapper package brms interfaced with Stan to perform sampling. ${ }^{30}$ 


\section{References}

1. Hopkins C, Fu SC, Chua E, Hu X, Rolf C, Mattila VM, et al. Critical review on the socio-economic impact of tendinopathy. Asia Pac J Sports Med Arthrosc Rehabil Technol. 2016; 4: 9-20.

2. Lewis J. Rotator cuff related shoulder pain: Assessment, management and uncertainties. Man Ther. 2016; 23:57-68.

3. Alizadehkhaiyat O, Fisher AC, Kemp GJ. Pain, functional disability and psychologic status in tennis elbow. Clin J Pain. 2007; 23(6): 482-489.

4. Seil R, Litzenburger H, Kohn D. Arthroscopic treatment of chronically painful calcifying tendinitis of the supraspinatus tendon. Arthroscopy. 2006; 22(5): 521-527.

5. Comin J, Malliaras P, Baquie P, Barbour T, Connell D. Return to competitive play after hamstring injuries involving disruption of the central tendon. Am J Sports Med. 2013; 41(1): 111-115.

6. Fu FH, Wang JH-C, Rothrauff BB. BMJ Best Practice Tendinopathy [Internet]. London: BMJ; 2019 [cited 2019 Jul 20]. Available from:

https://bestpractice.bmj.com/topics/en-gb/582

7. Albers IS, Zwerver J, Diercks RL, Dekker JH, Van den Akker-Scheek I. Incidence and prevalence of lower extremity tendinopathy in a Dutch general practice population: a cross sectional study. BMC Musculoskelet Disord. 2016; 17:16.

8. Littlewood C, May S, Walters S. Epidemiology of Rotator Cuff Tendinopathy: A Systematic Review. Shoulder \& Elbow. 2013; 5(4): 256-265.

9. Shiri R, Viikari-Juntura E, Varonen H, Heliövaara M. Prevalence and determinants of lateral and medial epicondylitis: A population study. Am J Epidemiol. 2006; 164(11): 1065-1074.

10. Vos T. Years lived with disability (ylds) for 1160 sequelae of 289 diseases and injuries 1990-2010: a systematic analysis for the global burden of disease study 2010. Lancet. 2012; 380(9859):2163-2196.

11. Kinge JM, Knudsen AK, Skirbekk V, Vollset SE. Musculoskeletal disorders in Norway: prevalence of chronicity and use of primary and specialist health care services. BMC Musculoskelet Disord. 2015;16(1):75.

12. Abat F, Alfredson H, Cucciarini M, Madry H, Marmott A, Mouton C, et al. Current trends in tendinopathy: consensus of the ESSKA basic science committee. Part I: biology, biomechanics, anatomy and an exercise-based approach. J Exp Orthop. 2017; 4:18.

13. Alfredson $H$. The chronic painful Achilles and patellar tendon: Research on basic biology and treatment. Scand J Med Sci Sports. 2005; 15(4): 252-259.

14. O'Neill S, Watson P, Barry S. Why are eccentric exercises effective for Achilles tendinopathy? Int J Sports Phys Ther. 2015; 10(4): 552-62.
15. Lim HY, Wong SH. Effects of isometric, eccentric, or heavy slow resistance exercises on pain and function in individuals with patellar tendinopathy: A systematic review. Physiother Res Int. 2018 Oct;23(4):e1721.

16. Young JL, Rhon DI, Cleland JA, Snodgrass SJ. The influence of exercise dosing on outcomes in patients with knee disorders: A systematic review. J Orthop Sports Phys Ther. 2018; 48(3): 146-161.

17. Wasfy MM, Baggish AL. Exercise dose in clinical practice. Circulation. 2016; 133(23): 2297-313.

18. Silbernagel KG. Does one size fit all when it comes to exercise treatment for Achilles tendinopathy? J Orthop Sports Phys Ther. 2014; 44(2): 42-44.

19. Meyer A, Tumilty S, Baxter GD. Eccentric exercise protocols for chronic non-insertional Achilles tendinopathy: How much is enough? Scand J Med Sci Sports. 2009; 19(5): 609-615.

20. Krämer R, Lorenzen J, Vogt PM, Knobloch K. Systematic review about eccentric training in chronic Achilles tendinopathy. Sportverletz Sportschaden. 2010; 24(4): 204-211.

21. Naunton J, Street G, Littlewood C, Haines T, Malliaras P. Effectiveness of progressive and resisted and nonprogressive or non-resisted exercise in rotator cuff related shoulder pain: A systematic review and meta-analysis of randomized controlled trials. Clin Reahbil. 2020; 34(9): 1198-1216.

22. Alexander et al. Exercise therapy for the treatment of tendinopathies: A scoping review protocol. JBI Evid Synth. 2021; doi: 10.11124/JBIES-20-00175.

23. United Nations Development Programme-Human Development Reports. Human development index: Table 1 human development index and its components 2020; Retrieved from: http://hdrundporg/en/composite/HDI.

24. Alfredson H, Pietilä T, Jonsson P, Lorentzon R. Heavy load eccentric calf muscle training for the treatment of chronic Achilles tendinosis. Am J Sports Med. 1998; 26(3): 360-366.

25. Cohen J. Weighted kappa: nominal scale agreement with provision for scaled disagreement or partial credit. Psychol Bull. 1968; 70: 213-220.

26. Higgins et al. The Cochrane Collaboration's tool for assessing risk of bias in randomised trials. BMJ. 2011; 343:d5928.

27. Sterne et al. ROBINS-I: A tool for assessing risk of bias in non-randomised studies of interventions. BMJ. 2016; 355:i4919.

28. Cochrane handbook 7.7.3. https://handbook-51.cochrane.org/chapter_7/7_7_3_data_extraction_for_con tinuous_outcomes.htm.

29. Marinho VCC, Higgins JPT, Logan S, Sheiham A. Fluoride toothpaste for preventing dental caries in children and adolescents. Cochrane Database of Systematic Reviews. 2003; 1:Art No: CD00227850.

30. Bürkner PC. brms: An R Package for Bayesian Multilevel Models Using Stan. I Stat Softw. 2017; 80(1): 28. 
Appendix I: Definitions use to define resistance treatment and treatment classes.

\begin{tabular}{|c|c|c|c|}
\hline Treatment Class & Definition & Treatment & Definition \\
\hline \multirow{5}{*}{ Resistance } & \multirow{5}{*}{$\begin{array}{l}\text { Exercise designed primarily to increase } \\
\text { strength of muscles by causing them to } \\
\text { produce substantive force against an } \\
\text { applied resistance which can take } \\
\text { several forms including the mass of the } \\
\text { body or its segments, isoinertial } \\
\text { resistance, elastic resistance, or strength } \\
\text { training equipment such as isokinetic } \\
\text { devices. In tendinopathy, the stimulus } \\
\text { may also be intended to provoke } \\
\text { tendon remodelling, reduce pain and } \\
\text { improve function. }\end{array}$} & Concentric Only & $\begin{array}{l}\text { Includes movements where force } \\
\text { produced overcomes the resistance } \\
\text { such that muscle shortening occurs. }\end{array}$ \\
\hline & & Eccentric Only & $\begin{array}{l}\text { Includes movements where force } \\
\text { produced is less than the resistance } \\
\text { such that controlled muscle } \\
\text { lengthening occurs. }\end{array}$ \\
\hline & & Concentric and eccentric & $\begin{array}{l}\text { Includes movements where force } \\
\text { produced exceeds the resistance in one } \\
\text { phase and is less than the resistance in } \\
\text { another such that controlled muscle } \\
\text { lengthening and shortening occurs. }\end{array}$ \\
\hline & & Isokinetic & $\begin{array}{l}\text { Uses specialised exercise equipment } \\
\text { such that the resistance is adjusted in } \\
\text { real-time to ensure joint angular } \\
\text { velocity remains constant. }\end{array}$ \\
\hline & & Isometric & $\begin{array}{l}\text { Includes muscular actions against a } \\
\text { resistance such that joint angle remains } \\
\text { constant. }\end{array}$ \\
\hline
\end{tabular}




\section{Appendix II: Outcome domains and example outcomes included in review.}

\begin{tabular}{|c|c|c|}
\hline Domain & ICON Definition & Example Tools \\
\hline Disability & $\begin{array}{l}\text { Composite scores of a mix of patient- } \\
\text { rated pain \& disability due to the pain, } \\
\text { usually relating to tendon-specific } \\
\text { activities/tasks }\end{array}$ & $\begin{array}{l}\text { VISA scales; DASH; quick DASH; SPADI; Patient-rated tennis- } \\
\text { elbow evaluation questionnaire; Constant Murley Score; WORC } \\
\text { (Western Ontario Rotator Cuff Index); AOFAS (American } \\
\text { Orthopaedic Foot \& Ankle Society); Roles and Maudsley score; } \\
\text { ASES (American Shoulder \& Elbow Surgeons Index; Tegner } \\
\text { activity score; Lysholm knee scale; Pain free function questionnaire; } \\
\text { Ankle activity score; Subjective elbow Value (SEV); Placzek score; } \\
\text { Shoulder disability questionnaire; International Knee } \\
\text { Documentation Committee form (IKDC); Penn Shoulder score } \\
\text { (university of Pennsylvania shoulder score) (PSS); Brief pain } \\
\text { inventory (BPI); UCLA Shoulder Rating Scale; FILLA - functional } \\
\text { index of leg and lower limb; Neer Shoulder Score; Nirschl phase } \\
\text { rating scale; American Shoulder and Elbow Surgeon's (MASES) } \\
\text { questionnaire; Mayo Elbow Performance Score (MEPS); Shoulder } \\
\text { rating questionnaire (SRQ) }\end{array}$ \\
\hline $\begin{array}{l}\text { Pain over a specified } \\
\text { time }\end{array}$ & $\begin{array}{l}\text { Patient-reported pain intensity over } \\
\text { period of time e.g. morning/night/24- } \\
\text { hours/1-week }\end{array}$ & VAS; NRS Painful days in 3 months \\
\hline $\begin{array}{l}\text { Pain without further } \\
\text { specification }\end{array}$ & $\begin{array}{l}\text { Patient asked about pain levels without } \\
\text { reference to activity or timeframe }\end{array}$ & VAS; NRS; Borg CR10 Scale; Pain status \\
\hline $\begin{array}{l}\text { Physical function } \\
\text { capacity }\end{array}$ & $\begin{array}{l}\text { Quantitative measures of physical tasks } \\
\text { (e.g. hops, times walk, single leg squat) } \\
\text { includes muscle strength }\end{array}$ & $\begin{array}{l}\text { Counter movement jump; One-leg triple hop; Single-leg decline } \\
\text { squat; Muscle strength measured by dynamometry (hand-held, } \\
\text { isokinetic); Repetition maximum; Manual muscle testing. }\end{array}$ \\
\hline
\end{tabular}


DOI | SportsR $x$ iv Preprint version 1

\section{Appendix III: Search strategy}

MEDLINE (EBSCoHost)

Search conducted on 27 April, 2020

\begin{tabular}{|l|l|l|}
\hline Search & Query & $\begin{array}{l}\text { Records } \\
\text { retrieved }\end{array}$ \\
\hline$\# 1$ & $\begin{array}{l}\text { MH exercise OR AB exercis* OR MH “isometric contraction” OR MH rehabilitation OR TX } \\
\text { eccentric OR TX concentric OR TX “heavy slow resistance" OR TX isokinetic }\end{array}$ & 362,722 \\
\hline$\# 2$ & $\begin{array}{l}\text { MH tendinopathy OR MH “shoulder injuries" OR MH tendons OR MH “tendon injuries” OR } \\
\text { TX tendin* OR TX tendon* OR MH bursitis OR AB bursitis OR MH “posterior tibial tendon } \\
\text { dysfunction" OR MH “shoulder impingement syndrome” OR AB "greater trochanteric pain } \\
\text { syndrome" }\end{array}$ & 96,490 \\
\hline$\# 3$ & $\# 1$ AND \#2 & 4,363 \\
\hline Limited to 1998 to present & \\
\hline
\end{tabular}




\section{Appendix IV: Extraction codebook}

\begin{tabular}{|c|c|c|c|}
\hline \multicolumn{2}{|c|}{ Column } & Heading & Description \\
\hline \multirow{23}{*}{ 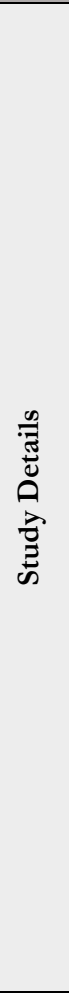 } & A & Initials Reviewer & Identification of individual extracting information \\
\hline & $\mathrm{B}$ & Covidence Identifier & Reference number for Covidence \\
\hline & $\mathrm{C}$ & Author & First author surname et al., \\
\hline & $\mathrm{D}$ & Year & Year of publication \\
\hline & $\mathrm{E}$ & Title & Study title \\
\hline & F & Country & Country where study was conducted \\
\hline & G & Journal & Journal name \\
\hline & $\mathrm{H}$ & Aims/Purpose & Study aims/purpose \\
\hline & $\mathrm{I}$ & Tendinopathy type & 1=Achilles; $2=$ Lateral elbow (tennis); $3=$ Patellar; $4=$ Rotator cuff (SI) \\
\hline & $\mathrm{J}$ & Study Design & RCT $=1 ;$ Quasi-experimental $=2$ \\
\hline & K & Age Mean & Mean age of study sample as a whole \\
\hline & $\mathrm{L}$ & Age SD & Standard deviation age of study sample as a whole \\
\hline & $\mathrm{M}$ & Baseline Total N & Total sample across all interventions measured at baseline \\
\hline & $\mathrm{N}$ & $\begin{array}{l}\text { Training Status } \\
\text { Description }\end{array}$ & Brief description of training status of study sample as a whole \\
\hline & $\mathrm{O}$ & Training Status Code & $1=$ Performance; $2=$ Sporting; $3=$ Other \\
\hline & $\mathrm{P}$ & Sex & Percentage female of study sample as a whole \\
\hline & Q & BMI Mean & Mean BMI of study sample as a whole \\
\hline & $\mathrm{R}$ & BMI SD & Standard deviation of BMI of study sample as a whole \\
\hline & $\mathrm{S}$ & Symptom Severity Mean & Mean severity measure at baseline of study sample as a whole \\
\hline & $\mathrm{T}$ & Symptom Severity SD & Standard deviation of severity measure at baseline of study sample as a whole \\
\hline & $\mathrm{U}$ & $\begin{array}{l}\text { Symptom Duration } \\
\text { Mean (Months) }\end{array}$ & Mean symptom duration reported in months \\
\hline & $\mathrm{V}$ & $\begin{array}{l}\text { Symptom Duration SD } \\
\text { (Months) }\end{array}$ & Standard deviation symptom duration reported in months \\
\hline & W & Population Comments & $\begin{array}{l}\text { Any additional information relevant to the participants investigated including } \\
\text { diagnostic criteria }\end{array}$ \\
\hline \multirow{4}{*}{$\underset{ٌ}{\mathscr{\Xi}}$} & $\mathrm{X}$ & Outcome Category & $\begin{array}{c}1=\text { Disability; } 2=\text { Pain on loading } / \text { activity; } 3=\text { Pain over a specified time; } 4= \\
\text { Pain without further specification; } 5=\text { Physical function capacity }\end{array}$ \\
\hline & & Outcome Tool & Description of outcome tool \\
\hline & $\mathrm{Z}$ & Reflection & $\begin{array}{c}1=\text { Increase in outcome indicates positive treatment; }-1=\text { Decrease in outcome } \\
\text { indicates positive treatment }\end{array}$ \\
\hline & AA & $\begin{array}{l}\text { Measurement Time } \\
\text { (Weeks) }\end{array}$ & Time of measurement in weeks \\
\hline \multirow{12}{*}{ 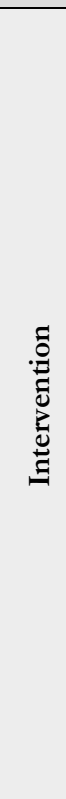 } & $\mathrm{AB}$ & Dominant Treatment & $\begin{array}{c}\text { Only one dominant theme to be selected } \\
1=\text { Concentric only; } 2=\text { Eccentric only; } 3=\text { Concentric and eccentric; } 4= \\
\text { Isokinetic; } 5=\text { Isometric }\end{array}$ \\
\hline & $\mathrm{AC}$ & Total Treatment & $\begin{array}{c}\text { Multiple themes to be selected as required } \\
1=\text { Concentric only; } 2=\text { Eccentric only; } 3=\text { Concentric and eccentric; } 4= \\
\text { Isokinetic; } 5=\text { Isometric }\end{array}$ \\
\hline & $\mathrm{AD}$ & Dose Comparison & $1=$ Lower dose intervention; $2=$ Higher dose intervention \\
\hline & $\mathrm{AE}$ & Intervention $\mathrm{N}$ & Intervention sample size at specified time \\
\hline & $\mathrm{AF}$ & $\begin{array}{l}\text { Intervention Total } \\
\text { Duration }\end{array}$ & Total duration of exercise intervention in weeks \\
\hline & AG & $\begin{array}{l}\text { Intervention Adherence } \\
\%\end{array}$ & Reporting of adherence to exercise (reported as a percentage) if applicable \\
\hline & $\mathrm{AH}$ & Intervention Location & $\begin{array}{l}\text { Location exercise was performed } \\
1=\text { Home; } 2=\text { Clinic } 3=\text { Fitness facility } 4=\mathrm{NR} ; 5=\mathrm{NA}\end{array}$ \\
\hline & $\mathrm{AI}$ & Intervention Volume & Numerical value describing volume \\
\hline & AJ & $\begin{array}{l}\text { Intervention Volume } \\
\text { Category }\end{array}$ & $\begin{array}{c}1=\text { Duration of session (mins); } 2=\text { sets } * \text { repetitions; } 3=\text { number of repetitions; } \\
4=\text { number of sets }\end{array}$ \\
\hline & $\mathrm{AK}$ & $\begin{array}{l}\text { Intervention Volume } \\
\text { Comments }\end{array}$ & Any additional information relevant. \\
\hline & $\mathrm{AL}$ & Intervention Intensity & Numerical value describing intensity \\
\hline & $\mathrm{AM}$ & $\begin{array}{l}\text { Intervention Intensity } \\
\text { Category }\end{array}$ & $1=$ Absolute; 2 = Relative \\
\hline
\end{tabular}


DOI | SportsR $x$ iv Preprint version 1

\begin{tabular}{|c|c|c|c|}
\hline & $\mathrm{AN}$ & Intervention Frequency & $\begin{array}{c}\text { Number of sessions per week. Where there is progression, average value is to be } \\
\text { entered. }\end{array}$ \\
\hline & $\mathrm{AO}$ & $\begin{array}{l}\text { Intervention Frequency } \\
\text { Comments }\end{array}$ & Any additional information relevant. \\
\hline & $\mathrm{AP}$ & $\begin{array}{l}\text { Intervention } \\
\text { Progression }\end{array}$ & $\begin{array}{c}\text { Multiple themes to be selected as required } \\
1=\text { No progression; } 2=\text { NR; } 3=\text { Progression volume; } 4=\text { Progression intensity; } \\
5=\text { Progression frequency; } 6=\text { Progression specificity; } 7=\text { Progression capacity; } \\
\quad 8=\text { Other }\end{array}$ \\
\hline & AQ & $\begin{array}{l}\text { Intervention } \\
\text { Progression Comments }\end{array}$ & Any additional information relevant. \\
\hline \multirow{5}{*}{$\stackrel{\pi}{\tilde{\pi}}$} & AR & $\begin{array}{l}\text { Intervention Baseline } \\
\text { Mean }\end{array}$ & Baseline mean for exercise therapy \\
\hline & AS & $\begin{array}{l}\text { Intervention Baseline } \\
\text { SD }\end{array}$ & Baseline standard deviation for exercise therapy \\
\hline & AT & $\begin{array}{l}\text { Intervention } \\
\text { Measurement Mean }\end{array}$ & Mean of outcome for exercise therapy at stated time point \\
\hline & $\mathrm{AU}$ & $\begin{array}{l}\text { Intervention } \\
\text { Measurement SD }\end{array}$ & Standard deviation of outcome for exercise therapy at stated time point \\
\hline & AV & $\begin{array}{l}\text { Measurement } \\
\text { Comments }\end{array}$ & $\begin{array}{l}\text { State if a different value has been entered for means (e.g. median), a different } \\
\text { value for standard deviations (e.g. standard error, IQR, percentiles, distance from } \\
\text { mean to upper bound). Provide the relevant statistic (width of CI's, width of } \\
\text { percentiles). Also state if data has extracted by digitization }\end{array}$ \\
\hline
\end{tabular}

* Outcome Specific 